\title{
A RELATIONSHIP BETWEEN THE BRIGHTNESS TEMPERATURES FOR TYPE III BURSTS
}

\author{
D. B. MELROSE*
}

Division of Radiophysics, CSIRO, Sydney, Australia

\begin{abstract}
Solar Phys.). The widely accepted emission mechanisms for type III bursts involve at least two stages. The first stage is the generation of Langmuir waves by the inferred stream of electrons. Emission at the fundamental frequency arises when these waves are scattered by thermal ions. Emission at the second harmonic arises when two Langmuir waves coalesce; however, the coalescence is possible only after an intermediate stage in which the distribution of Langmuir waves evolves towards isotropy due to scattering by thermal ions.

Quantitative theories for these emission processes are unsatisfactory because uncertainties in both stages compound each other. However, the predicted brightness temperatures $T_{1}$ and $T_{2}$, at the fundamental and the second harmonic respectively, at a given plasma frequency $f_{p}$ depends only on the spectrum of Langmuir waves and on coronal parameters. After making a plausible choice of the form of the spectrum of Langmuir waves, it is possible to eliminate the parameter describing their level of excitation (e.g. their energy density) to find a relation between $T_{1}, T_{2}$ and $f_{p}$. Comparison of this predicted relation with the observed quantities should avoid uncertainties associated with the theory of the generation of the Langmuir waves by the stream.
\end{abstract}

Theory predicts

with

$$
T_{1}=\left(T_{1}\right)_{0}\left(e^{\tau}-1\right), \quad T_{2}=\left(T_{2}\right)_{0} \tau^{2},
$$

$$
\left(T_{1}\right)_{0} \simeq 10^{9} \mathrm{~K}, \quad\left(T_{2}\right)_{0} \simeq \frac{10^{21} L_{2}}{f_{p}^{2} L_{1}^{2}} \mathrm{~K},
$$

and

$$
\tau \simeq 3 \times 10^{-7} W^{l} L_{1} / f_{p},
$$

where $f_{p}$ is in megahertz, $W^{l}$ is the energy density in Langmuir waves in ergs per cubic centrimetre, and $L_{1}$ and $L_{2}$ are the path lengths in centimetres over which generation of the fundamental and second harmonic respectively occur. In the above quantitative estimates the temperature of the corona has been taken as $10^{6} \mathrm{~K}$, the velocity of the stream $v_{s}$ and the velocity spread $\Delta v_{s}$ have been taken as $v_{s}=c / 3$ and $\Delta v_{s}=v_{s} / 3$ and the phase velocities of the Langmuir waves have been assumed to be in this same range. In the estimate of $T_{2}$ the Langmuir waves have been assumed isotropic; the

* On leave from Dept. of Theoretical Physics, Faculty of Science, The Australian National University, Canberra. 
characteristic time-scale on which they become isotropic is

$$
t \sim\left(3 \times 10^{11} W^{l} / f_{p}\right)^{-1} \mathrm{~s},
$$

where the units are as above. With $W^{l} \geq 3 \times 10^{-10}$ for $T_{1}>10^{9} \mathrm{~K}(\tau \geqslant 1)$ at $f_{p}=100$ MHz this gives $t \lesssim 1 \mathrm{~s}$, i.e. less than the duration of a type III burst (at fixed frequency at $100 \mathrm{MHz}$ ).

The characteristic lengths can be estimated from the bandwidth of the emission mechanisms and the characteristic distance $L_{N}=f_{p}\left|\operatorname{grad} f_{p}\right|^{-1}\left(\sim 3 \times 10^{10} \mathrm{~cm}\right.$ at $f_{p}=80 \mathrm{MHz}$ ) over which the plasma frequency changes. The bandwidth over which emission occurs is very narrow, e.g. $\Delta f / f \sim 10^{-3}$, at both the fundamental and the second harmonic. Because of this reasonable estimates would be $L_{1} \sim L_{2} \sim 3 \times 10^{7}$ to $10^{8} \mathrm{~cm}$.

Eliminating $\tau$ between the Equations (1) leads to a relationship between $T_{1}$ and $T_{2}$ for fixed $f_{p}$. This relationship is plotted in Figure 1 for $L_{1}=L_{2}=10^{8} \mathrm{~cm}$ and $f_{p}=$

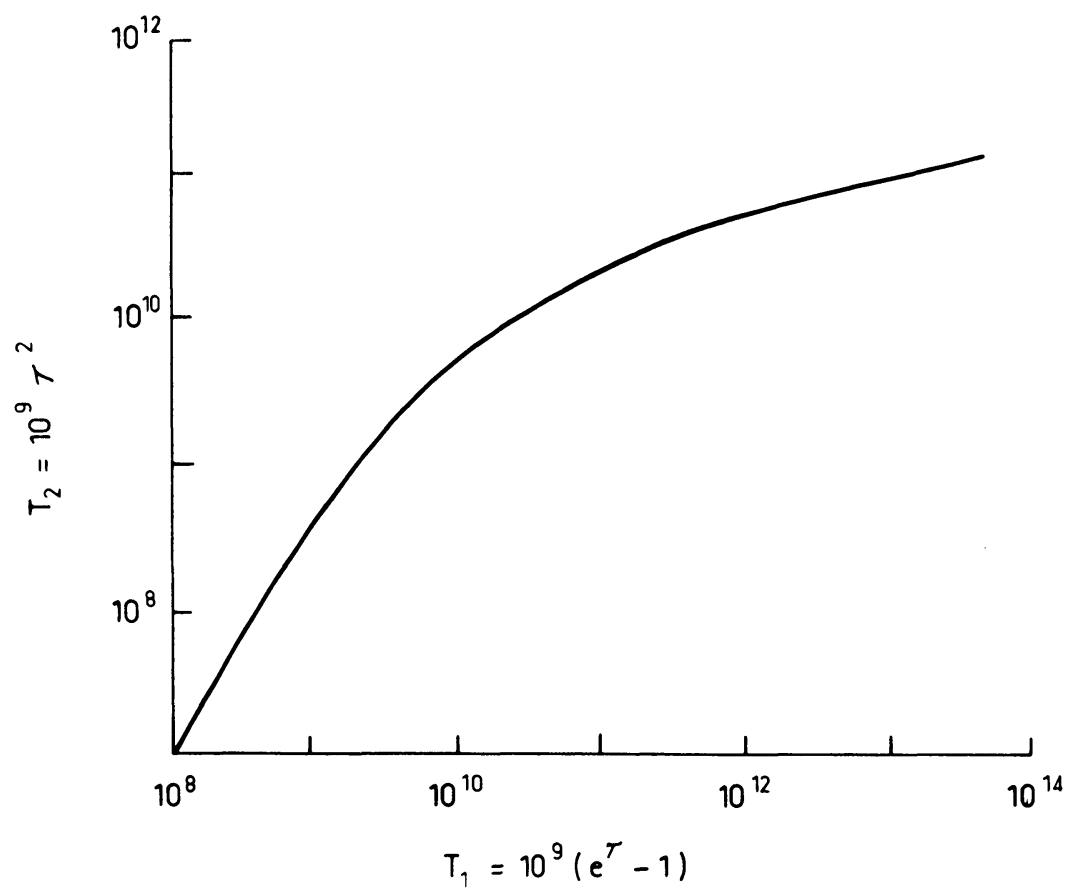

Fig. 1. The brightness temperature at the second harmonic $\left(T_{2}\right)$ is plotted as a function of the brightness temperature at the fundamental $\left(T_{1}\right)$ for $f_{p}=100 \mathrm{MHz}$ and $L_{1}=L_{2}+10^{8} \mathrm{~cm}$.

$=100 \mathrm{MHz}$. It should be emphasized that the relevant comparison is at fixed plasma frequency and not, for example, of the fundamental and second harmonic at a single frequency.

The relationship predicted here is the simplest one compatible with theory. It should be anticipated that the relation between the observed brightness temperatures will be found to deviate from the predicted relation. Various effects which have been 
neglected would favour specific kinds of deviation. Thus the qualitative form of the difference between the predicted and any observed relation should provide further insight into the detailed properties of the emission process.

\section{DISCUSSION}

Leblanc: If one wants to compare the brightness temperature of the fundamental and the harmonic emission, one must take into account scattering effects, since the fundamental suffers much more absorption than the harmonic.

Melrose: I agree. The effect you mention, namely collisional damping, favours the second harmonic. There are other effects, listed in my paper, some of which would favour the fundamental. The theory I have presented is the 'zeroth' order one. To improve on it we need to know whether it is consistent with observation and, if it is not, in which direction theory and observations differ.

Rosenberg: Wouldn't you expect that, if induced scattering plays an important role, the burst would be highly polarized?

Melrose: I would not say that I expect it or not, but it is possible. Dr Sy's paper later in the session is concerned with one mechanism whereby induced scattering leads to highly polarized radiation. 